\title{
Scrutinizing lasers
}

\author{
Lasers play a pivotal role in photonics, but claims of lasing are not always as robust and informative \\ as they should be. A new trial policy at Nature Photonics aims to rectify this shortcoming.
}

As a source of intense, coherent narrowband light, the laser undoubtedly plays a critical role in underpinning photonics research. It is not only an essential tool for many important scientific experiments that require photons but is also a convenient and efficient source of light at many wavelengths for technological

applications such as optical communications, materials processing, biomedical imaging and therapy, and environmental sensing, for example.

It is thus fair to conclude the origin of the field of photonics largely lies with the invention of the laser in 1960.

Today, almost 60 years later, despite an enormous number of different laser systems being demonstrated with media ranging from gases, liquids, crystals, semiconductors, fibres and even biological matter, research into lasers is still as active as ever. Researchers around the globe continue to strive to make lasers with unprecedented properties - be it nanolasers on new size scales, giant pulsed lasers with new levels of extreme peak power on the petawatt scale, or devices with new wavelengths of operation or evergreater efficiency.

While the physics of lasers dictates that they usually have a distinct signature of operation, concerns have been raised in the community that sufficient evidence to support a claim of lasing is not always provided in scientific literature ${ }^{1,2}$.

To address this issue, Nature Photonics has compiled and is launching a 'laser checklist' that authors of all manuscripts where a claim of lasing is the central result will be asked to complete prior to peer review. The aim is to ensure that common critical information related to the operation of the device (including a threshold behaviour, linewidth narrowing and a coherent output) is provided to help improve the reporting standards, level of transparency and aid reproducibility for papers on lasers. The information provided on the completed checklist will be shared with the reviewers and in the case of eventual publication of the paper be published alongside it as supplementary information. The checklist will initially be trialled for a period of 12 months and then continued, revised or stopped depending on the feedback received. The initial dialogue that we have had with the photonics community suggests that the initiative is likely to be well received, however.

"This is certainly an excellent idea.

The checklist will be very helpful to reviewers," commented Oliver Benson from Humboldt-Universität zu Berlin in Germany, who has published a wide variety of papers on lasers in the area of nanooptics and semiconductors.

\section{Nature Photonics is launching}

a 'laser checklist' that

authors of all manuscripts where a claim of lasing is the central result will be asked to complete prior to peer review.

"It was interesting to see you taking this initiative. I am generally supportive of it. Indeed, it can be very hard to prove laser action in novel situations. The interpretation of laser action has changed dramatically over the past 50 years or so," commented Rupert Oulton, an expert in plasmonics and nanolasers from Imperial College London, UK who provided valuable feedback on the checklist. "Expression of pumping conditions is very important. But more important still is that authors provide pump thresholds in power, current or energy density, that is, units should be $\mathrm{W} \mathrm{cm} \mathrm{cm}^{-2}, \mathrm{~A} \mathrm{~cm}^{-2}$ or $\mathrm{J} \mathrm{cm}^{-2}$. In this form, it is then easy to see how good a laser is. I am often frustrated that authors state W, A or J for thresholds, which is absolutely useless unless you know the area through which this value applies," concluded Oulton.

Steve Forrest, an expert in organic lasers, also welcomes the introduction of the checklist. He comments that care needs to be taken when measuring the linewidth of the emission, which should narrow at threshold if lasing is occurring, and that a capture of the emitted beam from the device is also useful.

"Linewidth narrowing should bring you to the $1 \AA$ level unless a spectrometer with inadequate resolution is used. If it is a multimode device, clear modal spectral resolution is required and the separation between modes should be determined by the cavity length. Here, poor spectral resolution is not to be tolerated," he commented. "The beam above threshold should show narrowing for the transverse mode that is being observed. As one of my grad students at one time (involved in our InGaAsP laser work) said so eloquently, 'No beam, no laser'. Beam images are really helpful, regardless of wavelength."

We appreciate that in certain circumstances it will not be feasible to provide data related to all the questions on the checklist but we trust that authors will be as diligent as possible. The checklist will shortly be available for download at http://www.nature.com/authors/policies/ laserchecklist.pdf. We hope that the optics community will embrace this new initiative and we welcome feedback on its further refinement.

\footnotetext{
References

1. Samuel, I. D. W., Namdas, E. B. \& Turnbull, G. A. Nat. Photon. 3, 546-549 (2009).

2. Kozlov, V. G., Bulović, V., Burrows, P. E. \& Forrest, S. R. Nature 389, 362-364 (1997)
}

\section{Acknowledgements}

We would like to specifically thank the following experts who all provided useful feedback related to the creation of the checklist and its contents. Ifor Samuels and Graham Turnbull from the University of St Andrews, UK, Steve Forrest at the University of Michigan, USA, Oliver Benson at Humboldt-Universität zu Berlin, Germany and Rupert Oulton from Imperial College London, UK. 